\title{
ANÁLISE DE IMAGENS HIPERESPECTRAIS PELO MÉTODO MULTIPLE ENDMEMBER SPECTRAL MIXTURE ANALYSIS (MESMA) EM DEPÓSITO SUPERGÊNICO DE NÍQUEL
}

\author{
OSMAR ABÍLIO DE CARVALHO JÚNIOR ${ }^{1}$, ANA PAULA FERREIRA DE CARVALHO², \\ PAULO ROBERTO MENESES ${ }^{3}$, RENATO FONTES GUIMARÃES ${ }^{4}$ \\ \& ÉDER DE SOUZA MARTINS ${ }^{5}$
}

\begin{abstract}
MULTISPECTRAL ANALYSIS BY THE MULTIPLE ENDMEMBER SPECTRAL MIXTURE ANALYSIS (MESMA) METHODS INA NICKEL SUPERGENE DEPOSIT The hyperspectral remote sensing provides a large amount of data allowing the development computing methods to improve detection and quantification of the materials that compose a given scene. In that context, the Multiple Endmember Spectral Mixture Models (MESMA) is an approach of the Spectral Mixture Analysis, which defines the best-fit model to describe each pixel. A key problem in this method is the computational time expended. The Spectral Correlation Mapper (SCM) was applied for a pre-classification of the material in order to decrease the computational time. The MESMA routine was developed in IDL language to identify the best-fit models considering the least RMS error. This method was applied to for the Airborne Visible/InfraRed Imaging Spectrometer (AVIRIS) subscene of Niquelândia, Goiás, that includes a lateritic nickel mine. The main minerals present in the weathering profile are: pimelite, saponite, goethite, hematite and kaolinite. The results attest that the calculated relative abundance of the mineral corresponds with field data.
\end{abstract}

Keywords: Spectral Mixture, MESMA, Imaging Spectroscopy.

Resumo O sensoriamento remoto hiperespectral, fornece grande quantidade de dados permitindo o desenvolvimento de métodos para detecção e quantificação dos materiais que compõem a cena. Neste contexto, o Multiple Endmember Spectral Mixture Models (MESMA) é uma evolução do método de análise linear de mistura que define o melhor modelo de mistura a ser utilizado para cada pixel. Um grande empecilho para a utilização desse método é o longo tempo computacional. O programa MESMA pode ser otimizado isolando-se os principais sistemas de misturas existentes na cena a partir do classificador espectral Spectral Correlation Mapper de forma a minimizar os membros finais e, conseqüentemente, diminuir de forma considerável o número de modelos e o tempo de processamento. Esta rotina foi desenvolvida em linguagem IDL identificando os melhores modelos a partir do menor erro médio quadrático. Esta metodologia foi aplicada na imagem Airborne Visible/InfraRed Imaging Spectrometer (AVIRIS) relativa à mina de Niquelândia, Goiás, com concentração supergênica de Ni-laterítico. Os principais minerais presentes no perfil de intemperismo são: pimelita, saponita, goethita, hematita e caulinita. Os resultados obtidos mostraram que a abundância relativa dos minerais está de acordo com os dados de campo.

Palavras-chave: Mistura Espectral, MESMA, Espectroscopia de Imageamento.

INTRODUÇÃO Uma nova concepção em processamento digital de imagens vem surgindo com o advento da espectroscopia de imageamento. A alta resolução espectral torna a informação de um dado pixel próxima à obtida por meio de medições espectrorradiométricas realizadas em laboratório e/ou campo (Abrams 1986, Vane \& Goetz 1988, Kruse 1988).

Desta forma, as imagens hiperespectrais representam uma nova abordagem com relação às técnicas de sensoriamento remoto para o uso geológico distinguindo-se, em certos aspectos, das utilizadas em imagens orbitais multiespectrais. Na concepção dessas imagens (MSS, TM-Landsat e SPOT), o principal enfoque foi a busca de técnicas de processamento digital de imagens com ênfase no realce visual de determinados alvos. Esses procedimentos geralmente promovem o realce da feição de interesse em detrimento das demais feições que compõem a cena. $\mathrm{O}$ advento dos sensores hiperespectrais estabelece uma mudança epistemológica no processamento digital de imagens ópticas. Essa tecnologia possibilitou avanços no estudo do pixel mistura. Apesar dos estudos baseados nessa abordagem terem origem na década de 70 (Horwitz et al. 1971, Richardson et al. 1975), o grande impulso para o seu desenvolvimento e aplicação veio a ser difundido com a espectroscopia de imageamento.

1 - INPE - Instituto Nacional de Pesquisas Espaciais, Avenida dos Astronautas 1758, 12201-970 - São José dos Campos - SP, E-mail: osmar@ltid.inpe.br 2 - Departamento de Ecologia, Universidade de Brasília, Campus Universitário Darcy Ribeiro, Asa Norte, 70910-900, Brasília, DF - E-mail: anapaula@unb.br

3- Instituto de Geociências, Universidade de Brasília, Campus Universitário Darcy Ribeiro, Asa Norte, 70910-900, Brasília, DF. E-mail: pmeneses@unb.br 4 - Departamento de Geografia, Universidade de Brasília, Campus Universitário Darcy Ribeiro, Asa Norte, , 70910-900, Brasília, DF. E-mail: renatofg@solar.com.br

5 - EMBRAPA Cerrados - Rodovia Brasília-Fortaleza, km 18, Planaltina, DF. E-mail: eder@cpac.embrapa.br 
O primeiro sistema de imageamento capaz de adquirir continuamente bandas ao longo da porção do espectro solar refletido, ou seja, de 0,4 a 2,5 mm foi o sensor AVIRIS (Airborne Visible/InfraRed Imaging Spectrometer), desenvolvido pela NASA (National Aeronautics and Space Administration), em 1983 (Vane et al. 1984) (Fig. 1). O desenvolvimento desse sistema teve como objetivo a obtenção de dados que pudessem ser utilizados nas diversas áreas das geociências. O AVIRIS tornou-se operacional em 1988 após alguns ajustes e correções realizadas pelos pesquisadores do JPL (Jet Propulsion Laboratory), NASA.

O AVIRIS foi trazido para o Brasil em 1995 na missão SCAR-B (Smoke, Clouds and Radiation - Brazil), que teve como propósito avaliar efeitos atmosféricos. Essa atividade foi uma operação conjunta da NASA, INPE (Instituto Nacional de Pesquisas Espaciais) e a AEB (Agência Espacial Brasileira) (Kaufman et al. 1998).

O sensoriamento remoto hiperespectral é uma tecnologia recente e em ampla expansão, o que proporciona uma constante proliferação de novos métodos e algoritmos para sua análise. Nessas imagens, a grande quantidade de informação permite uma ampla ramificação de métodos que visam otimizar e aprimorar a detecção e quantificação dos materiais que compõem uma cena.

ÁREA DE ESTUDO A área de estudo localiza-se no Maciço Máfico-Ultramáfico de Niquelândia, que contém importantes reservas de minério de níquel. As concessões de lavra dos depósitos de níquel estudados no presente trabalho pertencem à Companhia Níquel Tocantins, do Grupo Votorantim. Os jazimentos de níquel da Companhia Níquel Tocantins distam cerca de $23 \mathrm{~km}$ a norte da cidade de Niquelândia (Fig. 2). A estrada que liga a cidade de Niquelândia à usina de tratamento do minério é asfaltada. $\mathrm{O}$ acesso às minas é feito por estradas vicinais de terra.

Durante a missão SCAR-B, o sensor AVIRIS sobrevoou a região de Niquelândia no dia 16 de agosto de 1995. A linha de vôo foi realizada transversalmente ao complexo de Niquelândia, sobrevoando as unidades geológicas. A correção atmosférica da imagem foi feita pelo método de Green $(1990,1991)$, desenvolvido especificamente para as imagens hiperespectrais AVIRIS. Esse método proporciona uma estimava dos parâmetros atmosféricos e cálculo da reflectância aparente da superfície usando o código de transferência radiativa em conjunto com um modelo de ajuste não linear por mínimos quadrados (Green et al. 1991, 1993). Inicialmente, Green (1990) utilizou o código de transferência radiativa LOWTRAN (Keneizys et al. 1988), que foi, posteriormente, substituído por Green et al. (1993) pelo MODTRAN 3 (Berk et al. 1989, Anderson et al. 1995).

No presente trabalho é utilizada uma porção da imagem AVIRIS relativa à área da mina da Fazenda, principal mina em atividade da Companhia Níquel Tocantins. Por ser uma mina a céu aberto, extensa área é exposta evidenciando todos os horizontes do perfil de alteração.

Essa mina está sobre a subzona piroxenito-peridotítica apresentando-se, em muitos aspectos, compatível com a descrição da mina Jacuba de Colin et al. (1990). No entanto, apresenta algumas peculiaridades como mostra a descrição do perfil de intemperismo composto por: saprólito grosso, saprólito fino, saprólito fino ferruginoso, horizonte ferruginoso e a couraça silicosa (Fig. 3).

O Saprólito Grosso apresenta poucas exposições e, quando aflora, possui uma fina espessura, limitado apenas a um metro na

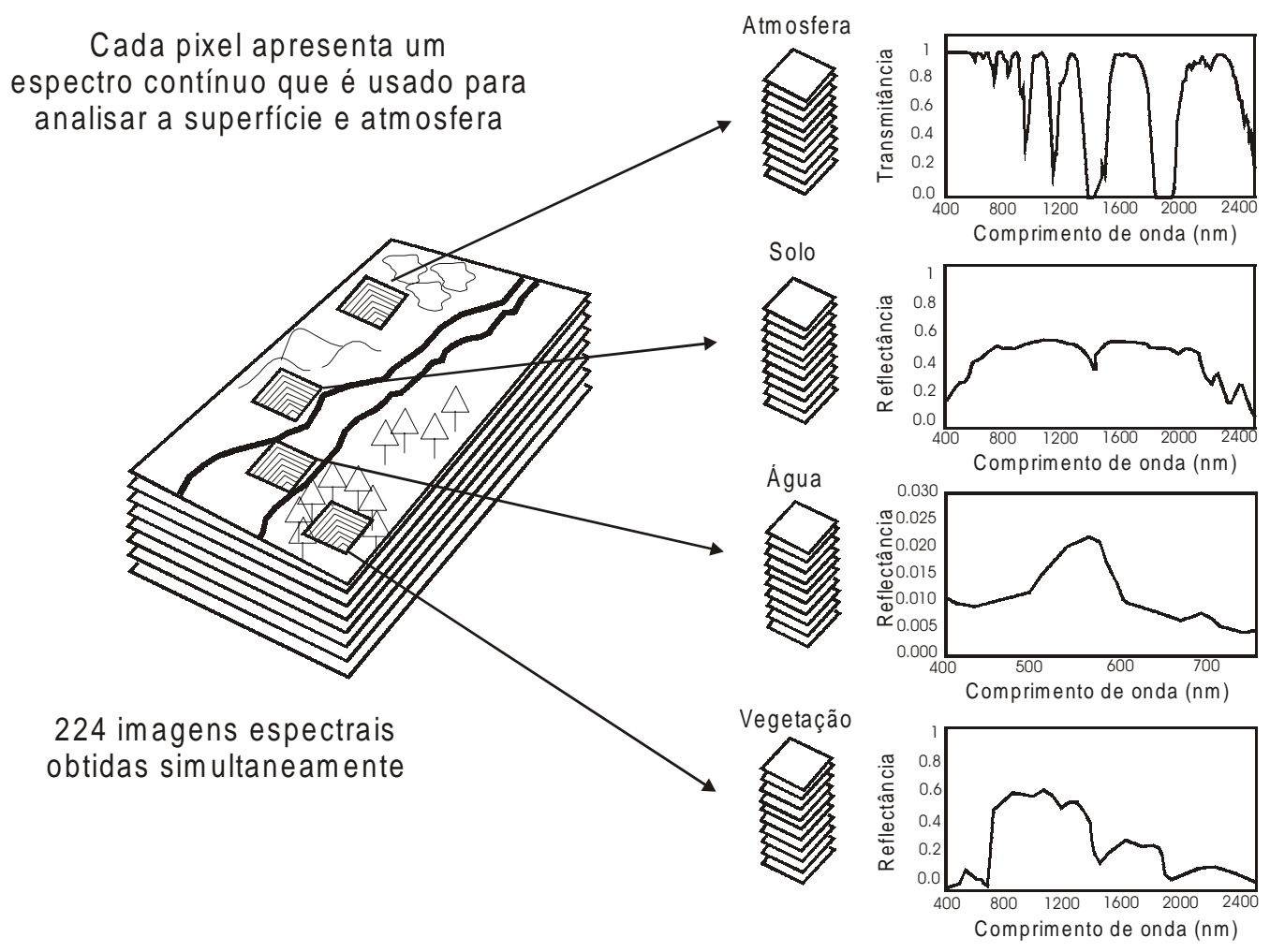

Figura 1 - Concepção do sensor hiperespectral AVIRIS: a alta resolução espectral torna a informação de cada pixel próxima à obtida por meio de medições realizadas em laboratório elou campo (Fonte: Green et al. 1998). 


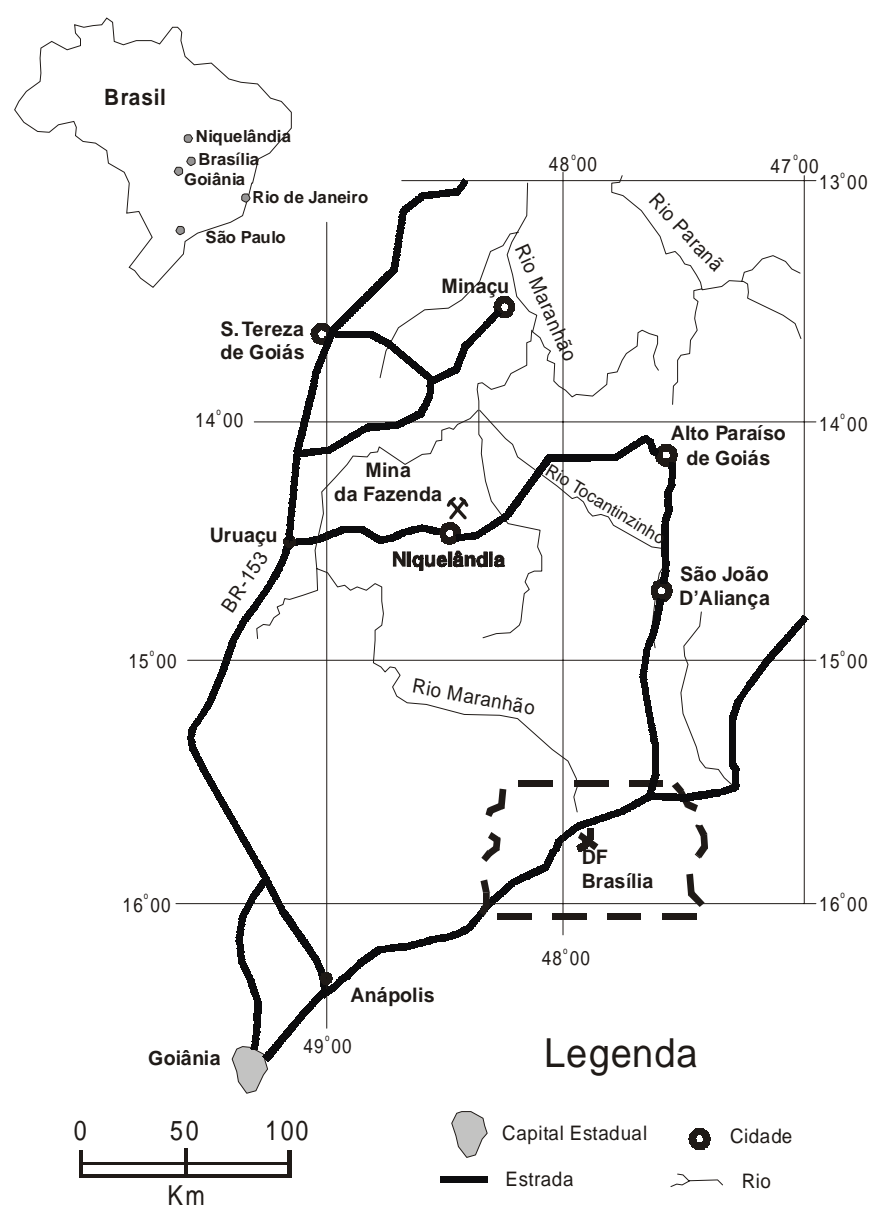

Figura 2 - Mapa de localização da região de Niquelândia

base do perfil, que o torna pouco representativo para a cava da mina. Caracteriza-se por um intenso padrão de fraturamento com espessura centimétrica a milimétrica preenchidas por esmectita. Ao longo das fraturas desenvolve-se a alteração do diopsídio e enstatita para esmectita.

O Saprolito Fino possui uma espessura de $10 \mathrm{~m}$ onde a base é constituída de material rico em pimelita, de coloração esverdeada, e para o topo, são observados Fe-saponita e oxi-hidróxidos de ferro, que dão uma coloração amarronzada. $\mathrm{O}$ contato com o saprólito grosso é enriquecido em veios garnieríticos.

O Saprólito Fino Ferruginoso possui espessura de $16 \mathrm{~m}$, sendo essencialmente goethítico e com estrutura maciça. Na base preserva a estrutura primária que é destruída progressivamente para o topo pelo aumento dos óxidos e hidróxidos de ferro que dominam a matriz. Apresentam fraturas preenchidas com asbolana (óxidos de Co-Mn-Ni). A transição da fácies silicatada para a oxidada ocorre em um intervalo de poucos metros. Todo o pacote apresenta-se dobrado, inclusive no contato. As dobras são abertas com um eixo de $\mathrm{N} 80^{\circ} \mathrm{W}$ e caimento variando de $30^{\circ}$ a $55^{\circ}$. Nos sinclinais ocorrem minerais oxidados, enquanto os anticlinais mantêm conservada a fácies silicatada. Desta forma, observam-se padrões de dobras abertas, onde domina a pimelita, nos anticlinais, e nos sinclinais, aumenta a proporção de óxido e hidróxido de ferro e Fesaponita.

O Horizonte Ferruginoso pode ser subdividido em três

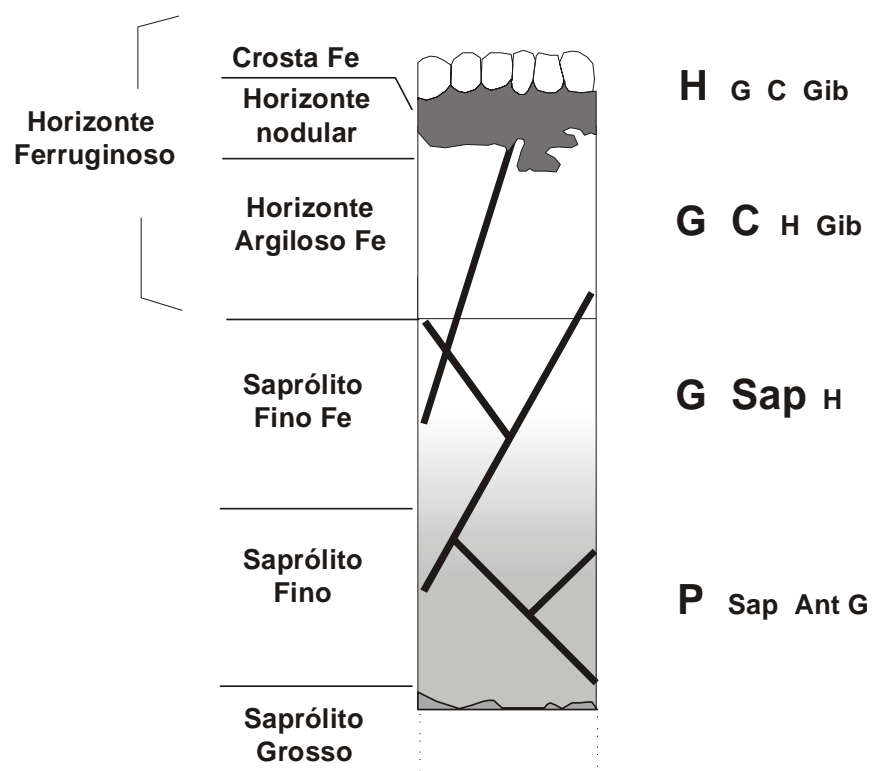

Figura 3 - Perfil de intemperismo da mina da Fazenda. $C=$ caulinita, $G=$ goethita, Gib = gibsita $H=$ hematita, Sap $=$ saponita, Ant = antigorita, $P=$ pimelita, letras maiúsculas = muita abundância, letras minúsculas = pouca abundância.

subhorizontes de forma similar ao perfil de Angiquinho descrito por Colin et al. (1990): Subhorizonte argiloso ferruginoso, Subhorizonte nodular e a crosta ferruginosa.

O Subhorizonte argiloso ferruginoso possui $10 \mathrm{~m}$ de espessura e apresenta estrutura terrosa fina dominada por goethita e caulinita, variando da cor avermelhada a esbranquiçada. A caulinita é abundante na parte superior do perfil, o que resulta em uma remoção do Ni devido à incompatibilidade com a estrutura deste argilomineral. Possui vênulas preenchidas por sílica. O Subhorizonte nodular possui espessura de $3 \mathrm{~m}$ e apresentam nódulos concrecionários goethíticos subarredondados, com granodecrescência ascendente variando de subcentimétrica a miliméticas. No topo do horizonte ferruginoso há uma couraça ferruginosa descontínua.

No topo do perfil há uma couraça silicosa alveolar, coberta por solo pouco espesso.

ANÁLISE LINEAR DE MISTURA (ALM) A análise linear de mistura baseia-se no emprego da regressão múltipla para quantificar a proporção dos minerais presentes na imagem. Esse procedimento teve sua origem no tratamento de imagens multiespectrais (Horwitz et al. 1971, 1975, Richardson et al. 1975, Adams \& Adams 1984, Huete 1986, Huete et al. 1986), obtendo grande propagação em estudos astronômicos com propósito de definir a composição superficial de astros (Singer \& McCord 1979, Pieters et al. 1985, Adams et al. 1986, Bell \& Hawke 1995, Blewett et al. 1995, Staid et al. 1996, Li et al. 1996, Mustard \& Head 1996, Smith et al 1985).

Com o advento do sensor Airborne Imaging Spectrometer (AIS) Smith \& Adams (1985b) transferiram e aprimoraram a metodologia para imagens hiperespectrais, denominando-a de Análise Linear de Mistura (ALM). Dentro dessa concepção foram realizados inú- 
meros trabalhos (Mustard \& Pieters 1986 1987, Mustard 1993, Roberts et al. 1993b).

Uma visualização da concepção do ALM pode ser obtida por meio da geometria do simplex (Boardman 1993). O simplex é uma forma geométrica que dispõe os pixels da imagem em um arranjo espacial que auxilia a compreensão das relações de mistura dos materiais. A disposição dos pixels em um espaço n-dimensional de atributo, cujos eixos não contém redundância de informação, como no caso da Análise de Principais Componentes (APC) e Minimum Noise Fraction (MNF), gera figuras geométricas que descrevem os membros finais e os produtos provenientes de sua mistura (Smith \& Adams 1985a, b, Bateson \& Curtiss 1993, 1996, Boardman 1993).

Esse conceito pode ser mais bem compreendido por meio de gráficos que representem o espaço de atributos. Para facilitar a representação é demonstrado um exemplo bi-dimensional onde os pontos relativos aos espectros estão distribuídos em forma triangular com relação à $1^{\mathrm{a}} \mathrm{e} 2^{\mathrm{a}}$ componentes MNF (Fig. 4).

Normalmente, verifica-se uma correlação da figura geométrica, gerada pela nuvem dos pixels, com o número de eixos presentes. Denota-se que, em um espaço $\mathrm{n}-$ dimensional, a nuvem de pixels forma uma figura que possui $(n+1)$ vértices, $(n+1)$ lados e $(n-1)$ dimensões, que é denominada de simplex. Desta forma, o simplex em 2-d é um triângulo com 3 vértices e 3 lados, enquanto que para um simplex 3-d é um tetraedro com 4 vértices e 4 lados e, assim, sucessivamente.

Todos os pontos do interior do simplex podem ser considerados como combinações dos seus vértices. Desta forma, o simplex, como retratação do espaço espectral, exibe nos seus vértices os pixels puros, enquanto os demais pixels contidos dentro dele são provenientes de suas misturas (Fig. 5). Portanto, a determinação dos membros finais requer a definição do melhor simplex que descreve os dados, seguida da identificação dos seus vértices.

Essa constatação permite descrever o processo de mistura do ponto de vista geométrico. Nesse âmbito, a forma mais usual de desmistura pelo simplex é realizada pela conversão de suas coordenadas para uma forma baricêntrica. Assim, todos os vértices ficam eqüidistantes do centro do simplex. A obtenção desse novo simplex é feita por um simples reescalonamento dos eixos que interligam os vértices para uma mesma dimensão, ou seja, todos os lados do simplex ficam iguais a 1 . Isso proporciona uma variação dos dados entre 0 e a unidade estabelecida. A figura 6 ilustra o procedimento, onde os vértices do simplex são linearmente transpostos para a forma de um simplex baricêntrico. Nesse novo arranjo, a posição de um determinado pixel determina a porcentagem existente de cada membro final. Esse procedimento coaduna com o método de análise linear de mistura proposto por Smith \& Adams (1985a, b). Pode-se descrever esse novo simplex com base
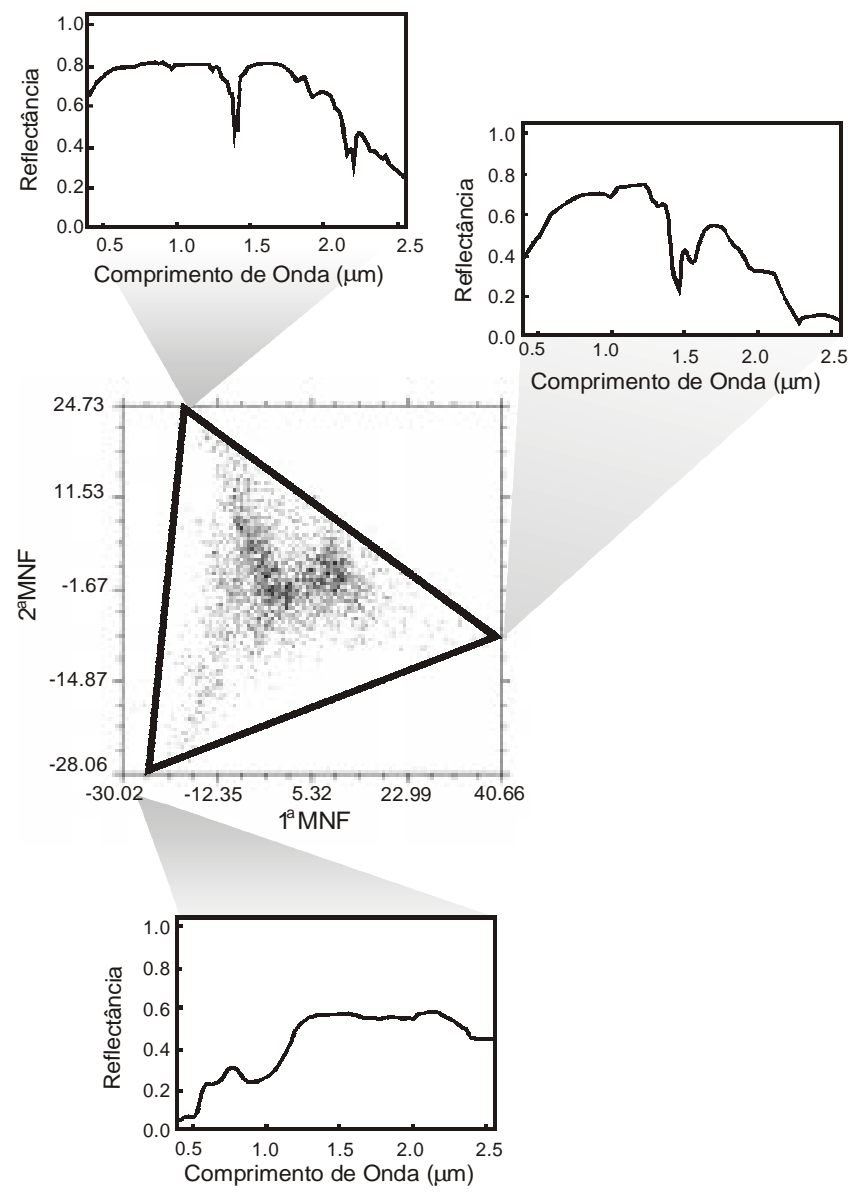

Figura 5 -Vértices do simplex indicando os membros finais ou os pixels mais puros.
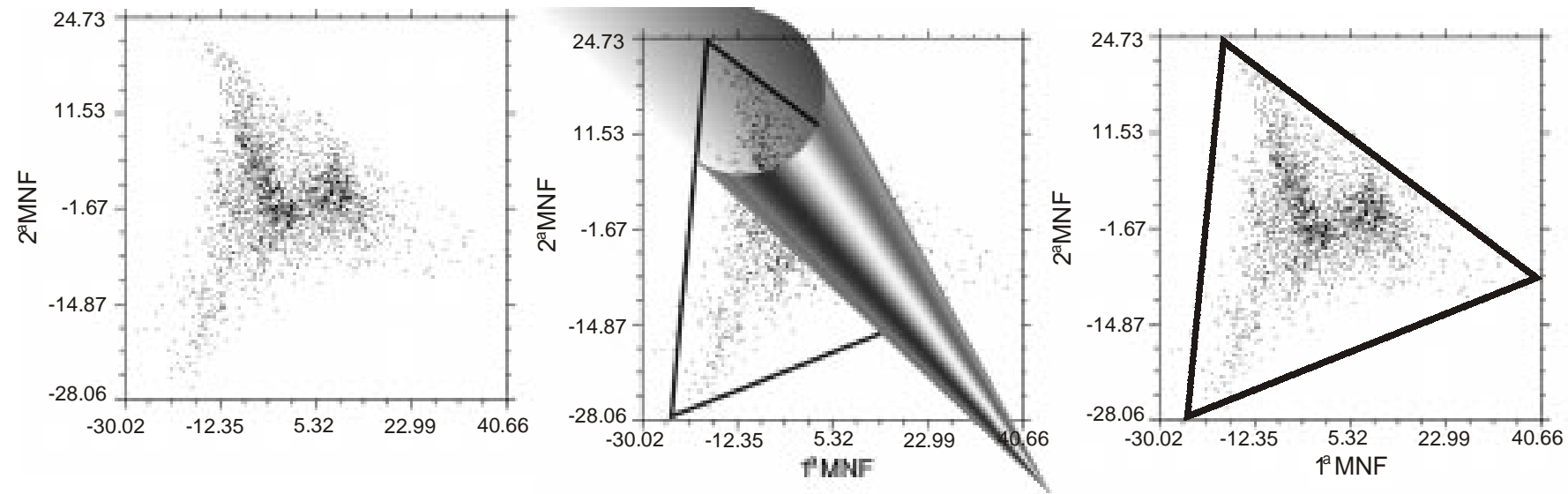

Figura 4 - Gráfico de dispersão com dados relativos à $1^{a}$ e $2^{a}$ componentes MNF apresentando uma configuração triangular. 
na ALM, de acordo com a seguinte expressão matemática:

$$
\begin{array}{ll}
\mathbf{R}_{\mathbf{b}}=\mathrm{Sf}_{\mathbf{i}} \mathbf{r}_{\mathrm{ib}}+\mathbf{E}_{\mathbf{b}} & \text { Equação 1 } \\
\mathrm{Sf}_{\mathrm{i}}=\mathbf{1 . 0} & \text { Equação 2 }
\end{array}
$$

onde: $\mathrm{R}_{\mathrm{b}}=$ reflectância do pixel na banda $\mathrm{b} ; \mathrm{f}_{\mathrm{i}}=$ fração de abundância do membro final i (a partir do total de membros finais); $r_{i b}=$ reflectância da banda $b$ do membro final $\mathrm{i} ; \mathrm{e}_{\mathrm{b}}=$ erro residual.

TIPOS DE ERROS DA ALM O êxito da desmistura dentro da configuração do simplex possui uma forte dependência da quantidade e qualidade dos membros finais utilizados. Como nesse procedimento há uma forte inter-relação de todos os dados, qualquer tipo de interferência por parte de um membro final afeta todos os pixels da cena analisada. Pode-se identificar três principais tipos de erros na ALM provenientes das características dos membros finais inseridos no modelo: a) retirada ou excessiva simplificação dos membros finais; b) inserção de muitos membros para descrever uma cena; e c) presença de espectros que não podem ser modelados a partir dos membros finais.

Retirada ou excessiva simplificação dos membros finais Quando um reduzido conjunto de membros finais é usado para descrever uma superfície complexa da imagem adicionam-se algumas incertezas na ALM. Sabol et al. (1992a) demonstraram o comportamento desse tipo de erro a partir de uma situação com quatro membros finais: a) granito, b) sombra, c) vegetação tipo 1 e d) vegetação tipo 2 . A exclusão da componente da vegetação tipo 2 ocasionou sua redefinição no simplex como sendo uma mistura dos demais elementos contendo aproximadamente $80 \%$ da vegetação 1, 15\% do granito e 5\% de sombra (Fig. 7). Portanto, toda vez que a vegetação tipo 2 estiver presente em um pixel, mesmo em pequenas quantidades, observa-se um deslocamento dos valores percentuais dos demais membros puros, como é indicado pelas setas da figura 7. Esse exemplo mostra como a retirada de um membro final pode resultar em erro de quantificação do elemento dentro da geometria do simplex.

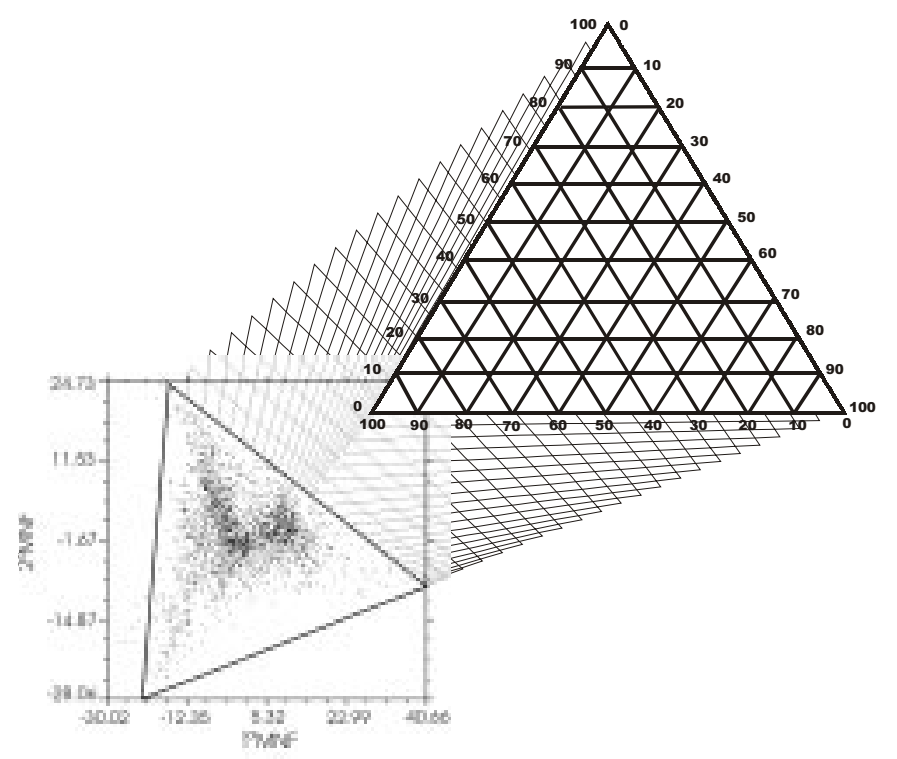

Figura 6 - Transformação do simplex para uma estrutura baricêntrica que permite estimar a proporção dos elementos de mistura.
Inserção de muitos membros finais para descrição de uma cena Cada membro final introduz no simplex um grau de erro inerente. Desta forma, na presença de muitos membros finais aumenta a nuvem de incerteza para uma estimativa (Fig. 8). Observa-se que o membro final com maior imprecisão ocasiona um alongamento da nuvem segundo sua direção. Na Figura 8 observam-se faixas de maior probabilidade de erro que aumentam em direção ao centro da elipse. Essa imprecisão pode levar a determinar para algum pixel a presença de um material que, na verdade, inexiste. Portanto, os principais fatores de erro com o aumento de membros finais são: a) aumento da variabilidade do erro; e b) estabelecimento de uma estimativa equivocada.

Presença de Espectros que Não Podem Ser Modelados Muitos

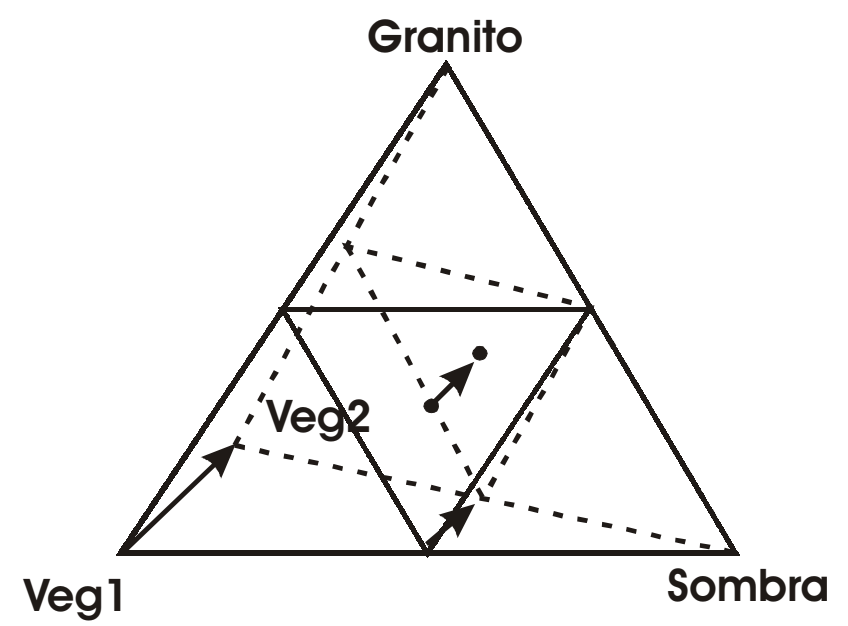

Figura 7 - Demonstração do erro pela não consideração do membro final referente à vegetação tipo 2. As setas indicam a alteração quando a vegetação tipo 2 está presente (Fonte: Sabol al. $1992 a$ - modificado).

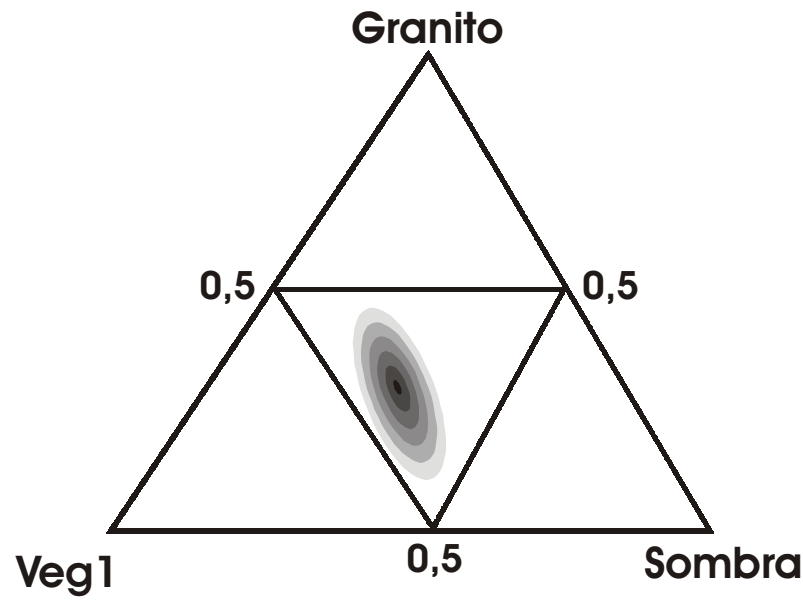

Figura 8-Demonstração da área de imprecisão referente aos diferentes membros finais (Fonte: Sabol et al. 1992amodificado). 
componentes que estão presentes em baixos teores na mistura não são passíveis de serem modelados adequadamente dentro do simplex. Esses, quando adicionados no modelo, provocam um aumento do erro. Tais elementos são denominados resíduos e devem receber tratamento especial. Como indicativos da presença de erro no procedimento ALM tem-se: a) aumento da incerteza do fracionamento; e b) aumento do residual ou do ruído.

\section{ANÁLISE DOS ERROS DA ALME NOVAS PROPOSIÇÕES} METODOLÓGICAS Tanto a insuficiência quanto o excesso de membros finais desencadeiam erros na ALM. Conclui-se, portanto, que é necessário atingir uma condição ideal de membros finais. Os melhores resultados são obtidos quando a cena possui poucos membros finais conciliados a um baixo índice de ruído.

No entanto, a utilização do ALM para cenas com uma inerente complexidade recai em uma das três fontes de erro anteriormente descritas. Nesses casos, cria-se um impasse, pois tanto a colocação de todos os membros finais quanto sua simplificação geram erros. Para contornar esse problema foram propostos outros métodos variantes da ALM que podem ser classificados em dois tipos metodológicos: a) o primeiro emprega a ALM com membros finais fixos para toda a imagem e realiza um estudo complementar do espectro residual, nessa categoria estão os métodos: Análise do resíduo, Análise Foreground - Background (AFB) e Análise Hierárquica do Foreground - Background (AHFB) (Gillespie et al. 1990, Smith et al. 1994, Pinzón et al. 1995), e b) o segundo emprega uma ALM específica para cada espectro da imagem com membros finais próprios que variam de pixel para pixel, nessa categoria tem-se o método denominado Multiple Endmember Spectral Mixture Analysis (MESMA) (Roberts et al. 1998a).

Análise do Resíduo Smith et al. (1987) e Gillespie et al. (1990) propuseram uma análise adicional ao modelo de desmistura que leva em consideração não somente as propriedades espaciais pertinentes ao simplex, como também as propriedades espectrais incluindo as feições de absorção. Esse modelo baseia-se na análise do resíduo, não modelado pela ALM, que retrata as feições de absorção de minerais com menores proporções na mistura e, conseqüentemente, de maior dificuldade de detecção. Observa-se no estudo do resíduo uma forte influência da análise das propriedades espectrais, desenvolvida por Clark \& Roush (1984) e Clark et al. (1990). Na análise de resíduo, a curva obtida pela ALM passa a comportar-se como uma linha para a remoção do contínuo. A metodologia consiste de duas etapas: a) ALM para os constituintes principais que compreende a cena, e b) análise dos resíduos para as feições não modeladas que representam bandas de absorção isoladas e discretas.

Esse método de caráter dual, que avalia tanto as características espectrais como espaciais, teve grande divulgação (Sabol et al. 1990, 1991,1992a, 1993, Roberts et al. 1991, 1993a). No entanto, apresenta dificuldades no estabelecimento de critérios para a distinção de alguns alvos entre membros finais e resíduos. Determinados alvos podem apresentar abundância variável na imagem, dificultando esta decisão. Além disso, a existência de mais de dois sistemas distintos de mistura na cena em análise, como por exemplo, a existência de área urbana e área natural, causa interferências e erros na quantificação, pois os membros finais de um sistema não são compatíveis com o outro.

Modelo de Análise foreground-background (AFB) Esse modelo foi proposto por Smith et al. (1994) e caracteriza-se por: a) realçar o material foreground, minimizando a variabilidade espectral para o conjunto de membros finais; e b) maximizar o contraste entre of foreground e o restante dos materiais presentes, denominados de background. A equação da AFB é:

$$
\begin{aligned}
& \text { Foreground } 1=\sum_{b=1}^{N b} W_{b} D N_{b}+c \text { Equação } 3 \\
& \text { Background } 0=\sum_{b=1}^{N b} W_{b} D N_{b}+c \text { Equação } 4
\end{aligned}
$$

onde o vetor $\mathbf{W}$ é definido como a projeção no hiperespaço que torna todos os níveis digitais do foreground iguais a 1 e do background iguais a $0 . \mathbf{N}_{\mathbf{b}}$ é o número de bandas e cé uma constante. A AFB suprime o efeito topográfico e minimiza o efeito da atmosfera e das nuvens.

Modelo de Análise Hierárquica do foregraound-backgraound Pinzón et al. (1995) modificaram a equação de análise do foreground - background (Smith et al. 1994) de forma a obter vários níveis hierárquicos. $\mathrm{O}$ procedimento permite determinar, a cada passo, a variabilidade dos dados, estabelecendo uma refinada extração das feições de absorção.

Esse modelo foi utilizado para a discriminação de solos e de suas propriedades em bacias hidrográficas das montanhas de Santa Mônica (Palacios-Orueta \& Ustin 1998, Palacios - Orueta et al. 1998, Pinzón et al. 1998) e de propriedades bioquímicas de folhas referentes à área de proteção ambiental de Jasper Ridge (Pinzón et al. 1995, 1998).

Modelo de Análise de Mistura Espectral Com Múltiplos Membros Finais (Multiple Endmember Spectral Mixture Analysis MESMA) A Análise Linear de Mistura falha por considerar todos os pixels como mistura de um único conjunto inicial de membros finais. Desta forma, pode-se ter um pixel modelado por membros finais que nele sejam inexistentes. Considerando-se uma cena descrita pelos membros finais: vegetação, solo e sombra, o cálculo da mistura será realizado com base nos três elementos, mesmo que não haja um dos componentes para o pixel.

Apesar da AFB e AHFB permitirem uma análise de feições existentes nos espectros que não foram inicialmente modeladas pelos membros finais, são métodos limitados quando a variabilidade de integrantes na imagem é alta. Esses métodos ainda dependem de uma escolha inicial correta dos membros finais, que muitas vezes é impossibilitada pela presença de diferentes ambientes de mistura e variabilidade alta dos membros finais escolhidos. Portanto, apesar de procurarem contornar os erros da ALM com uma segunda etapa de análise do resíduo, ainda recaem em muitos dos erros anteriormente apontados para a ALM.

Para corrigir essa fonte de erro foi proposto um novo método que estabelece para cada pixel o melhor modelo de mistura e assim realiza o cálculo de abundância dos membros finais (Smith et al. 1992, Sabol et al. 1992b, Roberts et al. 1992). A evolução dessa proposta deu origem ao modelo de Análise de Mistura Espectral com Múltiplos Membros (Multiple Endmember Spectral Mixture Analysis - MESMA) (Roberts et al. 1998a). O MESMA difere do ALM por permitir que o número e o tipo de membros finais, tanto quanto sua abundância, variem de pixel para pixel.

O MESMA utiliza bibliotecas formadas por espectros de campo que estabelecem, por análise combinatória dos membros finais, todos os modelos de mistura possíveis para cada pixel. Para cada 
modelo de um determinado pixel define-se: a) fração dos membros finais, b) a raiz do erro médio quadrático, e c) o residual.

A determinação do modelo de mistura mais adequado para um determinado pixel é obtida pelos critérios de menor valor residual e menor erro médio quadrático. Assim, o melhor modelo com seus valores de abundância são determinados pela combinação linear dos membros finais de tal forma que se obtenha o menor erro médio quadrático, quando comparado com a curva espectral do pixel.

O peso dos coeficientes varia de 1 até 0 e o ajuste é restrito para um limite de erro médio quadrático aceitável. Modelos que apresentam essa condição são registrados como um dos possíveis modelos para um determinado pixel. Como passo final, o modelo com menor erro médio quadrático para cada pixel pode ser identificado (Okin et al. 1998). O método gera como produto imagens de abundância dos membros finais e dos modelos de mistura. $\mathrm{O}$ MESMA tem sido aplicado no mapeamento de áreas com cobertura de neve (Painter et al. 1998a, 1998b) e vegetação (Roberts et al. 1998a, 1998b, Okin et al. 1998, 1999a).

OTIMIZAÇÃO DO MESMA OMESMAé um aperfeiçoamento da ALM, mais robusto, que permite amenizar os erros de forma considerável. Um grande empecilho para a utilização desse método é o longo tempo computacional exigindo o emprego de supercomputadores, o que torna seu uso bastante restrito (Okin et al. 1999b). O tempo operacional do MESMA está intimamente ligado à quantidade de membros finais que descreve uma cena e o número de modelos a serem comparados para cada pixel. Para propor métodos que otimizem o procedimento é importante compreender as relações existentes entre os membros finais e seus graus de interações.

Em uma imagem podem-se estabelecer vários sistemas de mistura que inter-relacionam-se entre si. Esses sistemas podem ser determinados pelo grau de associação da mistura. Em um primeiro enfoque pode-se buscar os principais membros que compõem a imagem como: solo, vegetação e rocha. Em uma segunda etapa pode-se determinar a abundância dos minerais que compõem o solo ou a rocha e a quantidade de folhas e ramos que compõem a vegetação.

Portanto, uma imagem pode ser descrita por um arranjo composto por vários simplex individualizados pelo grau da associação. Quanto maior for o grau de associação entre os membros de uma mistura, mais individualizado torna-se o seu simplex. Assim, as áreas vegetadas podem ser descritas por um simplex relativo a folhas verdes, folhas secas e galhos, que apresentam alto grau de associação, enquanto que o solo pode ser descrito pelos minerais hematita, caulinita e gibsita e assim, sucessivamente. Por sua vez, esses sistemas de misturas relacionam-se entre si em um outro simplex.

No programa MESMA a definição do melhor simplex de um pixel é obtida pelos modelos gerados a partir de todos os membros finais das diferentes associações de mistura. Assim, no caso de três associações compostas por três membros ter-se-ia nove membros finais gerando um total de 511 modelos para cada pixel a ser testado. Este procedimento gera muitos modelos com membros finais que afetam apenas alguns grupos de pixels, mas não têm a menor influência sobre os demais pixels da cena em análise, o que torna o processo moroso.

O programa MESMA pode ser otimizado isolando os principais sistemas de misturas existentes na cena de forma a minimizar os membros finais e, conseqüentemente, diminuir de forma conside- rável o número de modelos e o tempo de processamento. A determinação dos ambientes de mistura pode ser obtida pelo emprego de classificadores espectrais que comparam o espectro do pixel com um espectro de referência, proveniente de bibliotecas espectrais ou de membros finais. A comparação é realizada por meio de um critério de similaridade que caracteriza cada método. Os principais classificadores espectrais são: o Spectral Angle Mapper-SAM (Kruse et al. 1992, Kruse et al. 1993a e b), o Spectral Feature Fitting - SFF (ENVI 1997), o Binary Encoding (Mazer et al. 1988), o coeficiente de correlação R utilizado pelo Tricorder (Clark \& Swayze 1995) e o Spectral Correlation Mapper-SCM (Carvalho Júnior \& Meneses 2000). O SCM apresenta vantagens sobre os demais métodos devido à capacidade de detectar falsos positivos.

Desta forma, no presente trabalho é proposta uma simplificação do modelo MESMA a partir do emprego do SCM para distinguir os principais sistemas de mistura. A delimitação espacial dos sistemas de mistura pelo SCM é realizada por máscaras. Sobre as áreas pré-selecionadas realiza-se o cálculo de ALM para todas as possíveis combinações de mistura espectral. Esses modelos de ALM são agrupados em uma única imagem e submetidos a um programa desenvolvido em IDL que analisa pixel a pixel o melhor modelo de mistura, considerando o menor valor da raiz do erro médio quadrático. Os modelos de mistura com valores negativos para um determinado mineral são desconsiderados pelo programa.

APLICAÇÃODOMÉTODOMESMAEMNIQUELÂNDIA Para a determinação dos espectros de reflectância ao longo do perfil laterítico utilizou-se um espectrorradiômetro da marca GER (Geophysical \& Environmental Research Corp.), modelo IRIS (Infrared Intelligent Spectroradiometer) MARK V. Este instrumento opera na faixa espectral de 300 a $2500 \mathrm{~nm}$, com larguras de bandas de $2 \mathrm{~nm}$ no intervalo de 300 a $1000 \mathrm{~nm}$ e de $4 \mathrm{~nm}$, entre 1000 e $2500 \mathrm{~nm}$. Os espectros foram reamostrados de acordo com as características do sensor AVIRIS para facilitar sua posterior comparação com a imagem e a biblioteca espectral da USGS (Clark et al. 1993). Para complementar a análise espectral foi realizada uma análise mineralógica por difratometria de raio X. Os principais minerais identificados que compõem o perfil de intemperismo são: pimelita, saponita, goethita, hematita, caulinita cujos espectros radiométricos são fornecidos na figura 9 .

A pimelita e a saponita estão contidas na garnierita que é um termo genérico para definir uma mistura de hidrossilicatos de magnésio e níquel (Trescasses et al. 1980, Brindley \& Pham 1973). Maquet et al. (1981) identificaram nas garnieritas da Nova Caledônia (mistura de serpentina e tipos intermediários de talco - saponita) feições espectrais de transições eletrônicas de campo cristalino decorrentes da presença do íon $\mathrm{Ni}^{2+}$ em coordenação octaédrica em 0,39mm, 0,66 mm 1,100 mm. Cervelle \& Maquet (1982) apresentaram uma leve alteração das duas primeiras feições para garnieritas, passando a considerar os valores de $0,38 \mathrm{~mm} \mathrm{e} 0,67 \mathrm{~mm}$. Porto (1994) identificou dois tipos de garnieritas para o complexo máfico - ultramáfico de Barro Alto: uma com predomínio de serpentina e a outra de talco. O comportamento espectral dessas garnieritas apresentava as feições do Fe e Ni descritas por Maquet et al. (1981).

A garnierita da Mina da Fazenda é constituída principalmente por pimelita, apresentando feições de transição eletrônica em $0,61 \mathrm{~mm}$ e, aproximadamente, em $1,10 \mathrm{~mm}$. Não foi possível detectar a feição da garnierita em 0,39 mm devido à limitação do instrumen- 

Níquel

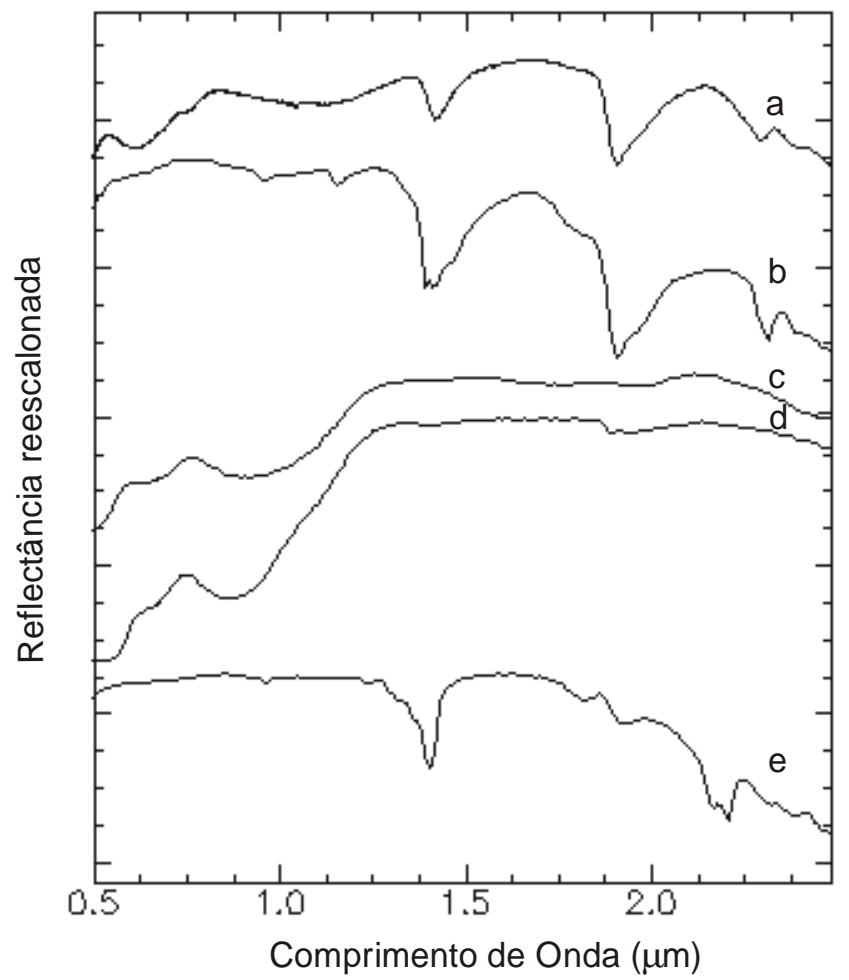

Figura 9- Curvas espectrais relativas aos minerais presentes na mina da Fazenda: a) pimelita, b) caulinita, c) goethita, d) hematita e e) saponita. As curvas $b, c, d$, e são da biblioteca da USGS (Clark et al. 1993).

to, mas pode-se avaliar sua tendência no espectro. A presença de magnésio na estrutura da pimelita gera feições de absorção devido às vibrações da ligação $\mathrm{Mg}-\mathrm{OH}$ posicionadas em $2,29 \mathrm{~mm}$ e $2,385 \mathrm{~mm}$. Garnieritas mais ricas em saponita apresentam essa feição em $2,31 \mathrm{~mm}$. As feições relativas ao magnésio apresentam vantagens na identificação dos minerais por não sofrerem interferências das feições provenientes do ferro como na região do visível.

A goethita e a hematita são discriminadas pelas feições do $\mathrm{Fe}^{3+}$. Sherman \& Waite (1985) descreveram as feições espectrais da hematita provenientes de transições eletrônicas de campo cristalino em $0,445 \mathrm{~mm}, 0,53 \mathrm{~mm}, 0,65 \mathrm{~mm}$ e $0,885 \mathrm{~mm}$, as quais se diferenciam da goethita que apresenta feições de absorção em $0,435 \mathrm{~mm}$, $0,48 \mathrm{~mm}, 0,65 \mathrm{~mm}$ e $0,917 \mathrm{~mm}$. Muitos autores utilizam o deslocamento das feições espectrais do $\mathrm{Fe}^{3+}$ para determinar a proporção relativa desses minerais (Towensend 1987, Formaggio et al. 1996, Kruse et al. 1990, Madeira Netto 1991, Madeira Netto et al. 1997). A caulinita é identificada pela dupla feição de absorção proveniente da ligação Al-OH em 2,2 mm (Hunt et al. 1971).

O MESMA foi utilizado na área de estudo com uma prévia seleção dos minerais pimelita, saponita, goethita, hematita e caulinita pelo método SCM. No emprego do SCM não é necessário preocupar-se com os limites exatos, podendo-se inclusive superestimar as áreas de abrangência, pois o MESMA encarrega-se de definir o melhor modelo.

Desta forma, foram delimitadas na área da mina as localidades com exposição do solo e do perfil de intemperismo (Fig. 10). As demais áreas excluídas pela máscara apresentam-se cobertas por vegetação e sem interesse para a detecção mineral. Com o propósito de facilitar a compreensão da distribuição mineral na imagem, a figura 10 apresenta a localização da parte mais baixa da mina a céu aberto onde está o minério silicatado, a parte do topo da mina onde está o minério oxidado, as estradas de acesso e a pilha de minério.

$\mathrm{Na}$ análise mineral constatou-se que o MESMA apresentou um melhor desempenho considerando apenas a intervalo espectral relativo as feições de absorção dos minerais em análise. Portanto foram utilizados a faixas espectrais entre $0,43 \mathrm{~mm} \mathrm{a} 0,75 \mathrm{~mm}$ e $2,09 \mathrm{~mm}$ a 2,38mm.

A figura 11 apresenta as imagens resultantes para cada mineral onde as áreas mais claras correspondem às maiores porcentagens do mineral. As imagens relativas aos minerais são concordantes com os resultados observados no campo. Uma topossequência a partir da base da cava da mina para o topo é bem caracterizada pela imagem. A base do perfil é constituída por pimelita e goethita e, à medida que se dirige para o topo, observa-se um aumento da quantidade de hematita e caulinita. Observa-se uma predominância da pimelita na mina e da saponita na pilha. A imagem da raiz do erro médio quadrático mostra que as áreas com garnierita foram as que apresentaram os maiores valores de erro, provavelmente devido à dificuldade de ajuste na porção do visível.

No programa desenvolvido também é gerada uma imagem relativa aos melhores modelos definidos pelo método MESMA. A figura 12 mostra os dez modelos detectados na área que configura a imagem, sendo os demais desprezados. Os modelos para a cava da mina apresentam-se coerentes. Eventualmente pode-se observar alguns modelos de mistura que não condizem com o perfil, como a presença de pimelita na área de topo da mina. Isto pode ser atribuído aos movimentos dos caminhões que trazem o minério silicatado para a porção superior. Esses pixels apresentam quantidade de pimelita bastante reduzida, como mostra a figura 13.

CONCLUSÃO O método Multiple Endmember Spectral Mixture Analysis (MESMA) é um aperfeiçoamento do método Análise Linear de Mistura (ALM) que possibilita identificar o modelo de mistura existente para cada pixel. Como o método requer muito

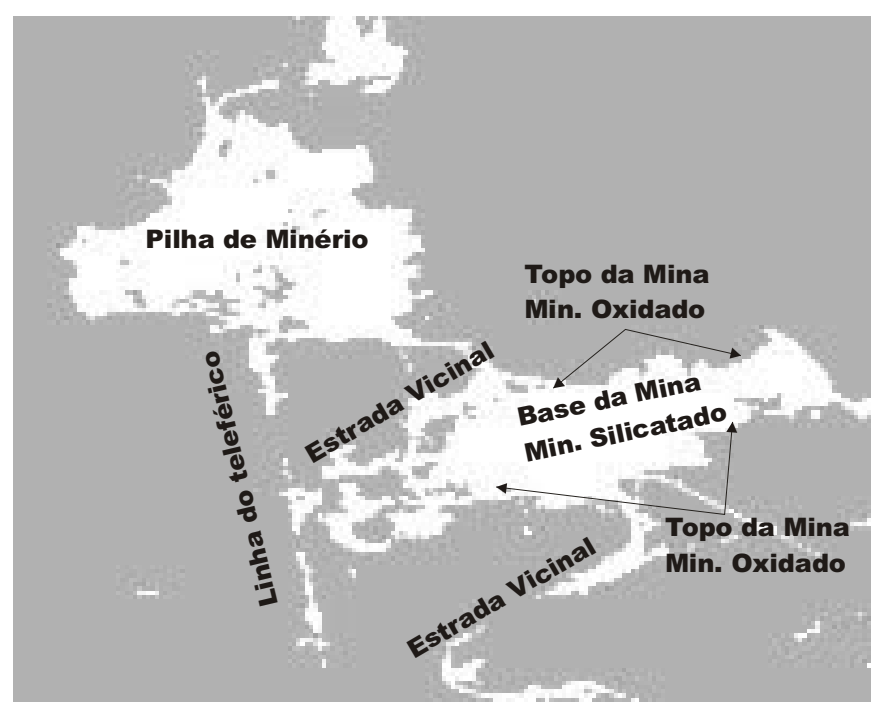

Figura 10 - Máscara com delimitação das áreas com exposição do solo e perfil de intemperismo (regiões claras). 

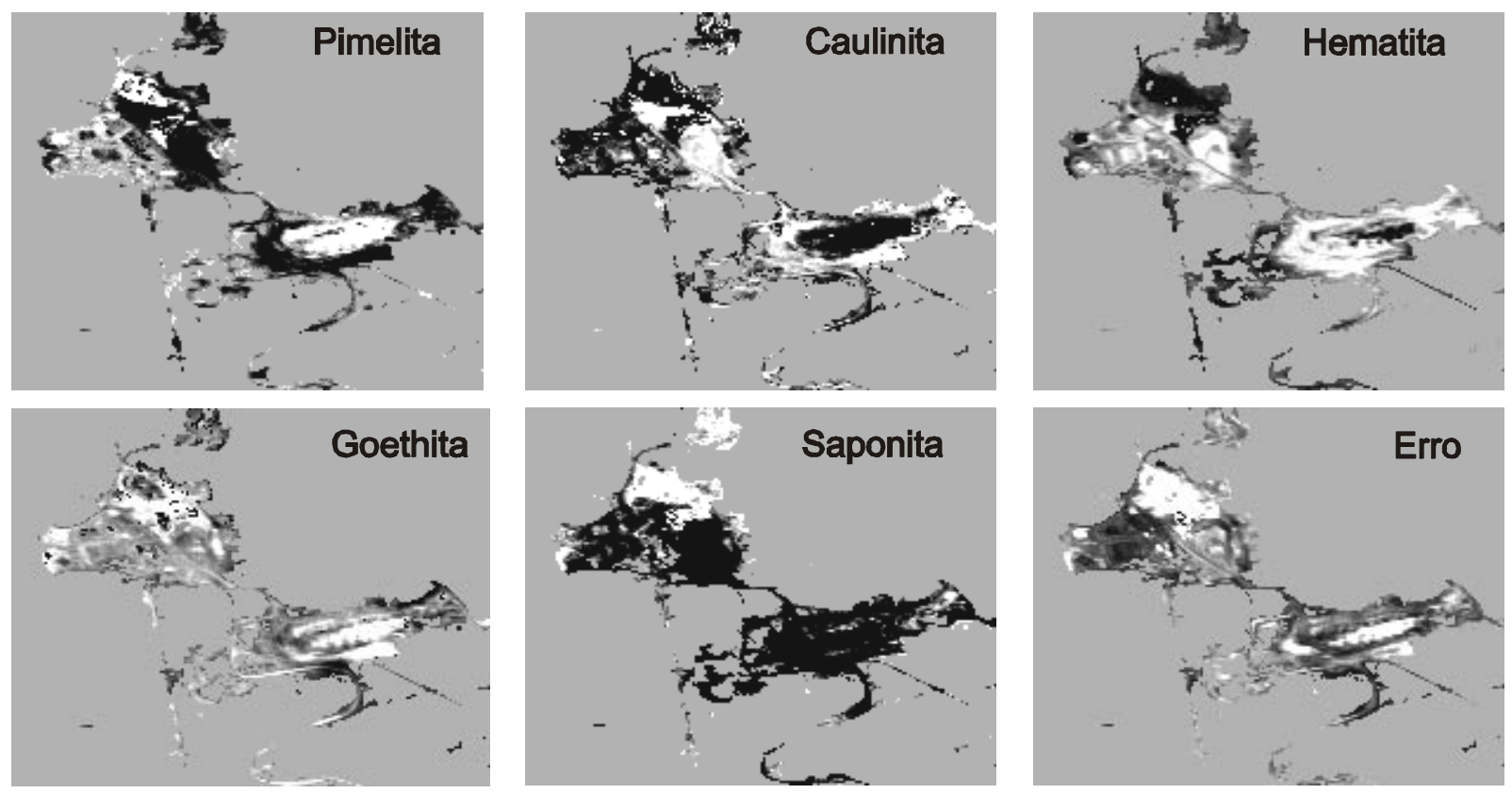

Figura 11 - Imagens do método MESMA para pimelita, caulinita, hematita, goethita, saponita e raiz do erro médio quadrático.
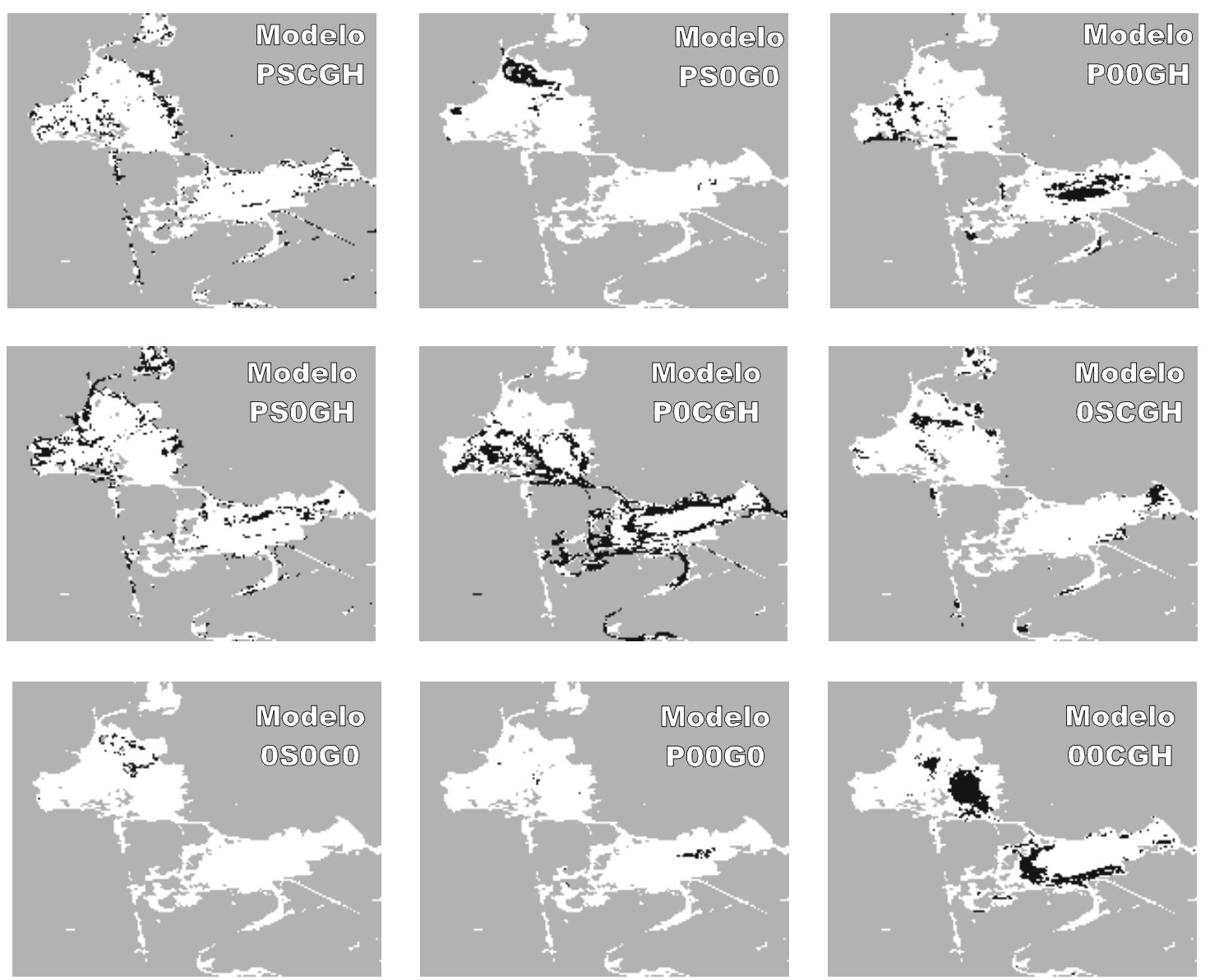

Figura 12 - Principais modelos de mistura selecionados pelo MESMA salientados em preto. Os minerais presentes no modelo estão representados pelas letras maiúsculas (P-pimelita, S-saponita, C-caulinita, G-goethita, H-hematita e 0 - inexistência do mineral). 
tempo operacional, uma forma para torná-lo mais ágil é o emprego de uma prévia seleção das áreas de interesse utilizando um classificador espectral, como o Spectral Correlation Mapper (SCM). Cabe ressaltar que os limites das áreas de abrangência, classificados pelo SCM, não precisam ser exatos, podendo ser superestimados, pois o MESMA encarrega-se de definir o melhor modelo.

O MESMA obtém melhores resultados quando são utilizados apenas as faixas relativas às feições de absorção existentes nos minerais analisados. O MESMA é um método completo, pois tanto permite identificar as áreas de ocorrência de minerais como quantificar a sua relativa abundância.

Agradecimentos Aos revisores da RBG pelas sugestões aos manuscrito.

\section{Referências}

Abrams M.G. 1986. Imaging spectrometry: aircraft and space program. In: Int. Geosci. Remote Sensing Symp., Zurich, ESA, Proceedings, 231-234.

Adams J.B. \& Adams J. 1984. Geologic mapping using Landsat MSS and TM images: Removing vegetation by modeling spectral mixtures. In: Thematic Conf. Remote Sens. for Expl. Geol. ERIM, 3, Proceedings, 2:615-622.

Adams J.B., Smith M.O., Johnson P.E. 1986. Spectral mixture modeling. A new analysis of rock and soil types at the Viking Lander 1 Side. $J$. Geophys. Res. 91:8-98-8122.

Anderson G.P., Wang J., Chetwynd J.H. 1995. MODTRAN3: An update and recent validations against airborne high resolution interferometer measurements. In: Annual JPL Airborne Earth Science Workshop, 5, Pasadena, CA, Summaries, JPL Publ. 95-1, 5-8.

Bateson C.A. \& Curtiss B. 1993. A tool for manual endmember selection and spectral unmixing. In: Annual JPL Airborne Geoscience Workshop, 4, Pasadena, CA, Summaries, JPL Publ. 93-26, 3-6.

Bateson C.A. \& Curtiss B. 1996. A method for manual endmember selection and spectral unmixing. Remote Sens. Environ. 55:229-243.

Bell J.F. \& Hawke B.R. 1995. Composional variability of the Serenitatis/ Tranquillitatis region of the moon from telescopic multispectral imaging and spectroscopy. Icarus, 118:51-68.

Berk A., Bernstein L.S., Robertson D.C. 1989. MODTRAN: A moderate resolution model for LOWTRAN7. Final report, GL-TR-0122, AFGL, Hanscomb AFB, MA, 42pp.

Blewett D.T., Hawke B.R., Lucey P.G., Taylor G.J., Jaumann R., Spudis P.D. 1995. Remote sensing and geologic studies of the Schiller-Scickard region of the moon. J. Geophys. Res., 100:16959-16972.

Boardman J.W. 1993. Automated spectral unmixing of AVIRIS data using convex geometry concepts: In: Annual JPL Airborne Geoscience Workshop, 4, Pasadena, CA, Summaries, JPL Publ. 93-26, 11-14.

Brindley G.W. \& Pham T.H. 1973. The nature of garnierites: I. Structure chemical composition and color characteristics. Clays and Clays Mineral., 21:17-40.

Carvalho Júnior O.A. \& Meneses P.R. 2000. Spectral Correlation Mapper (SCM): an improving Spectral Angle Mapper. In: Annual JPL Airborne Earth Science Workshop, 9, Pasadena, CA, Proceedings, JPL Publ. 00-18, 65-74.

Cervelle B.D. \& Maquet M. 1982. Cristallochimie des lizardites substituees Mg-Fe_Ni par Spectrometrie Visible et Infrarouge Proche.

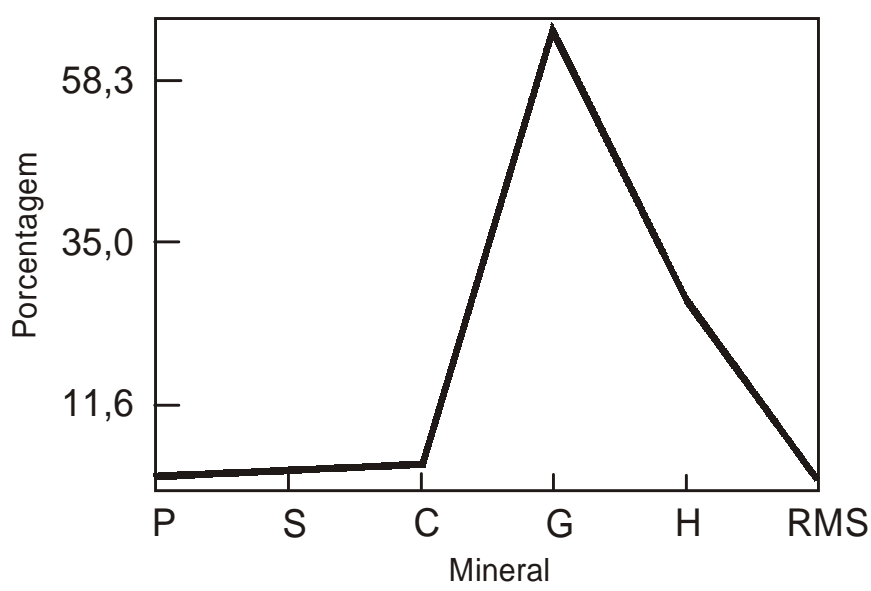

Figura 13 - Porcentagem relativa entre os minerais em um pixel no topo do perfil intempérico (P-pimelita, $S$-saponita, $C$ caulinita, G-goethita, H-hematita, RMS-raiz do erro médio quadrático).

Clay Min., 17:377-392.

Clark R.N. \& Roush T.L. 1984. Reflectande spectroscopy: quantitavive analysis techniques for remote sensing applications. J. Geophys. Res., 89:6329-6340.

Clark R.N. \& Swayze G.A. 1995. Mapping minerals, amorphous materials, environmental materials, vegatation, water, ice and snow, and other materials: the USGS Tricorder algorithm. In: Annual JPL Airborne Earth Science Workshop, 5, Pasadena, CA, Summaries, JPL Publ. 95-1, 39-40.

Clark R.N., Gallagher A.J., Swayze G.A. 1990. Material absorption band depth mapping of imaging specrometer data using a complete band shape least-squares fit with library reference spectra. In: Annual JPL Airborne Visible / Infrared Imaging Spectrometer Workshop, 2, Pasadena, CA, Summaries, JPL Publ. 90-54, 176-186.

Clark R.N., Swayze G.A., King T.V.V., Gallagher A.J., Calvin W.M. 1993. The U.S. Geological Survey, Digital Spectral Reflectance Library: version 1: 0.2 to $3.0 \mathrm{~mm}$. In: Annual JPL Airborne Geoscience Workshop, 4, Pasadena, CA, Summaries, JPL Publ. 9396, 37-38.

Colin F., Nahon D., Trescases J.J., Melfi A.J. 1990. Lateritic weathering of pyroxenites at Niquelandia, Góias, Brazil: the supergene behavior of nickel. Econ. Geol., 85:1010-1023.

ENVI®. 1997. Tutorials better solutions. Consulting Limited Liability Company Lafayette, Colorado, USA, 370pp.

Formaggio A.R., Epiphanio J.C.N., Valeriano M.M., Oliveira J.B. 1996. Comportamento espectral (450-2500nm) de solos tropicais de São Paulo. Rev. Bras. Ciência do Solo, 20:467 - 474.

Gillespie A.R., Smith M.O., Adams J.B., Willis S.C., Fischer A.F., Sabol D.E. 1990. Interpretation of residual images: spectral mixure anaysis of AVIRIS images, Owens Valley, California. In: Annual JPL Airborne Visible/Infrared Imaging Spectrometer (AVIRIS) Workshop, 2, Pasadena, CA, Summaries, JPL Publ. 90-54, 243-270.

Green R.O. 1990, Retrieval of reflectance from calibrated radiance imagery measured by the Airborne Visible/Infrared Imaging Spectrometer (AVIRIS) for lithological mapping of Clark Mountains, California. In: Annual JPL Airborne Visible/Infrared Imaging Spectrometer (AVIRIS) Workshop, 2, Pasadena, CA, Summaries, JPL Publ. 9054, 167-175. 
Green R.O. 1991. Retrieval of reflectance from AVIRIS - measured radiance using a radioative transfer code. In: Annual JPL Airborne Visible/Infrared Imaging Spectrometer (AVIRIS) Workshop, 3, Pasadena, CA, Summaries, JPL Publ. 91-28, 200-210.

Green R.O., Conel J.E., Margolis J.S., Bruegge J., Hoover G.L. 1991. An inversion algorithm for retrieval at atmospheric and leaf water absorption from AVIRIS radiance with compensation for atmospheric scattering. In: Annual JPL Airborne Visible/Infrared Imaging Spectrometer (AVIRIS) Workshop, 3, Pasadena, CA, Summaries, JPL Publ. 91-28, 51-61.

Green R.O., Conel J.E., Roberts D.A. 1993. Estimation of aerosol optical depth, and addicional atmospheric parameters for the calculation of apparent reflectance from radiance measured by the airborne visible/ infrared imaging spectrometer. In: Annual JPL Airborne Earth Science Workshop, 4, Pasadena, CA, Summaries, JPL Publ. 93-26, 83-86.

Green R.O., Eastwood M.L., Sarture C.M., Chrien T.G., Aronson M., Chippendale B.J., Faust J.A., Pavri B.E., Chovit C.J., Solis M., Olah M.R., Williams O. 1998. Imaging spectroscopy and the Airborne Visible/Infrared Imaging Spectrometer (AVIRIS). Remote Sens. Environ., 65:227-248.

Horwitz H.M., Lewis J.T., Pentland A.P. 1975. Estimating proportions of objects from multispectral scanner data. Final Report, NSAS Contract NAS9-14123, NASA-CR-141862, 108p.

Horwitz H.M., Nalepka R.F., Hyde P.D., Morgenstern J.P. 1971 Estimating the proportions of objects within a single resolution element of a multispectral scanner. In: Int'l Symp. Remote Sens. Environ. Ann Abor, 7, Proceedings, 1307-1320.

Huete A.R. 1986. Separation of soil-plant spectral mixture by factor analysis. Remote Sens. Environ., 19:237-251.

Huete A.R., Jackson R.D., Post D.F. 1986. Spectral response of plant cannopy with different soil backgrounds. Remote Sens. Environ., 17:37-53.

Hunt G.R., Salisbury J.W., Lenhoff C.J. 1971. Visible and near infrared spectra of minerals and rocks. III. Oxides and hydroxides. Modern Geology, 3:195-205.

Kaufman Y.J., Hobbs P.V., Kirchhoff V.W.J.H., Artaxo P., Remer L.A., Holben B.N., King M.D., Ward D.E., Prins E.M., Longo K.M., Mattos L.F., Nobre C.A., Spinhirne J.D., Ji O., Thompson A.M., Gleason J.F., Christopher S.A., Tsay S.-C.1998. Smoke, Clouds, and Radiation-Brazil (SCAR-B) experiment. J. Geophys. Res., 103(D24):31783-31808.

Kneizys F.X., Shettle E.P., Abreu L.W. 1988. Users guide to LOWTRAN7. AFGL-TR-8-0177, Air Force Geophys. Lab. Bedford, MA, 136p.

Kruse F.A. 1988. Use of Airborne Imaging Spectrometer data to map minerals associated with hidrothermally altered rocks in the Northern Grapevine Mountains, Nevada and California. Remote Sens. Environ., 24:31-52.

Kruse F.A., Kierein-Young K.S., Boardman J.W. 1990. Mineral mapping at Cuprite, Nevada with a 63-channel imaging spectrometer. Photogram. Engin. Remote Sens., 56:83-92.

Kruse F.A., Lefkoff A.B., Boardman J.W., Heiedbrecht K.B., Shapiro A.T., Barloon P.J., Goetz,A.F.H. 1992. The Spectral Image Processing System (SIPS) - software for integrated analysis of AVIRIS data. In: Annual JPL Airborne Geoscience Workshop, 3, Summaries, Pasadena, CA, JPL Pub-92-14, p 23-25.

Kruse F.A., Lefkoff B., Dietz J.B. 1993a. Expert System-Based Mineral Mapping in Northern Death Valley, California/Nevada, using the Airborne Visible/Infrared Imaging Spectrometer (AVIRIS). Remote Sens. Environ., 44:309-336.
Kruse FA, Lefkoff A.B, Boardman J.W., Heiedbrecht K.B., Shapiro A.T., Barloon P.J., Goetz A.F.H. 1993b. The Spectral Image Processing System (SIPS) - interactive visualization and analysis of imaging spectrometer data. Remote Sens. Environ. 44:145-163.

Li L., Mustard J.F., He G. 1996. Mixing across simple mare-highland contats: New insights from Clementine UV-VIS data of the Grimaldi basin. Lunar Planet. Sci., 27:751-752.

Madeira Netto J.S. 1991. Étude quantitative des relations constituants mineralogiques-réflectance difuse des latosols brésiliens/application al'utilisation pedologique des données satellitaires TM(regionde Brasilia). Thése de Doctorat, Université Pierre et Marie Curie. Paris. 224 p.

Madeira Netto J.S., Bédidi A., Cervelle B., Pouget M., Flay N. 1997. Visible spectrometric indices of hematite $(\mathrm{Hm})$ and goethtite $(\mathrm{Gt})$ content in lateritic soils: the application of Thematic Mapper (TM ) image for soil-mapping in Brasilia, Brazil. Int. J. Remote Sens., 18:2835-2852.

Maquet M., Cervelle B.D., Gouet G. 1981. Signatures of $\mathrm{Ni}^{2+}$ and $\mathrm{Fe}^{3+}$ in the optical spectra of limonitic ore from New Caledonia: Application to the determination of the nickel content. Mineral. Depos., 16:357-373.

Mazer A.S., Martin M., Lee M., Solomon J.E. 1988. Image processing software for imaging spectrometry analysis. Remote Sens. Environ., 24:201-210.

Mustard J.F. \& Head J.W. 1996. Highland mixing relationships along the Soutwestern Shores of Oceanus Procellarum. J. Geophys. Res., 101:18913-18925.

Mustard J.F. \& Pieters C.M. 1986. Quantitative abundance estimates from bidirectional reflectance measurements. J. Geophys. Res., E617E626.

Mustard J.F. \& Pieters C.M. 1987. Abundance and distribution of ultramafic microbreccia in Moses Rock dike: quantitative application mapping spectroscopy. J. Geophys. Res., 92:10376-10390.

Mustard J.F. 1993. Relationships of soil, grass, and bedrock over the Kaweah Serpentinite Melange through spectral mixture analysis of AVIRIS data. Remote Sens. Environ., 44:293-308.

Okin G.S., Okin W.J., Roberts D.A., Murray B. 1998. Multiple Endmember Spectral Mixure Analysis: application to an arid/semiarid landscape. In: JPL Airborne Earth Science Workshop, 7, Pasadena, CA, Summaries, JPL Publ. 97-21, 291-299.

Okin W.J., Okin G.S., Roberts D.A, Murray B. 1999a. Multiple Endmember spectral Mixture Analysis: Endmember choice in an arid shrubland. In: JPL Airborne Earth Science Workshop 8, Pasadena, CA, Summaries, JPL Publ. 99-17, 323-331.

Okin G.S., Siegel H., Collier J., Miller G.D., Okin W.J., Roberts D.A., Murray B., Curkendall, D.W., Painter T.H. 1999b. The supercomputing vizualization workbench for analysis and classification of imaging spectrometer data. In: JPL Airborne Earth Science Workshop, 8, Pasadena, CA, Summaries, JPL Publ. 99-17, 317-322.

Painter T.H., Roberts D.A., Dozier J., Green R.O. 1998a. Automated subpixel snow parameter mapping with AVIRIS data. In: JPL Airborne Earth Science Workshop, 7, Pasadena, CA, Summaries, JPL Publ. 97-21, 301-307.

Painter T.H., Roberts D.A., Green R.O., Dozier J. 1998b. The effect of grain size on spectral mixture analysis of snow - covered area from AVIRIS data. Remote Sens. Environ., 65:320-332.

Palacios-Orueta A. \& Ustin S.L. 1998, Remote Sensing of Soil Properties in the Santa Monica Mountains: I. Spectral Analysis. Remote Sens. Environ., 65:170-183 
Palacios-Orueta A, Pinzón J.E., Roberts D.A., Ustin S.L. 1998. Remote Sensing of Soils in the Santa Monica Mountains: Hierarchical Foreground and Background Analysis. In: JPL Airborne Earth Science Workshop, 7, Pasadena, CA, Summaries, JPL Publ. 97-21, 309-318.

Pieters C.M., Adams J.B., Mouginis-Mark P.J., Zisk S.H., Smith M.O., Head J.W., McCord T.B. 1985. The Nature of Crater Rays: The Copernicus example J. Geophys. Res., 90:12392-12413.

Pinzón J.E., Ustin S.L., Hart Q.J., Jacquemoud S., Smith M.O. 1995. Using foreground/background analysis to determine leaf and conopy chemistry. In: Annual JPL Airborne Visible/Infrared Imaging Spectrometer (AVIRIS) Workshop, 5, Pasadena, CA, Summaries, JPL Publ. 95-1, 129-132.

Pinzón J.E., Ustin S.L., Pierce J.F. 1998. Robust feature extraction for hyperspectral imagery using both spatial and spectral redundancies. In: JPL Airborne Earth Science Workshop, 7, Pasadena, CA, Summaries, JPL Publ. 97-21, 325-334.

Porto S.G. 1994. Comportamento espectral das lateritas niqueliferas do complexo máfico-ultramáfico de Barro Alto, Goiás. Universidade Federal do Rio Grande do Sul, Dissertação de Mestrado, 196 p.

Richardson A.J., Wiegand C.L., Gausman H.W., Cuellar J.A., Genermann A.H. 1975. Plant, soil and shadow reflectance components of row crops. Photogramm. Engr. Remote Sens., 41:1401-1407.

Roberts D.A., Smith M.O., Adams J.B., Gillespie A.R. 1991. Leaf spectral types residuals, and canopy shade in an AVIRIS image. In: Annual JPL Airborne Visible/Infrared Imaging Spectrometer (AVIRIS) Workshop , 3, Pasadena, CA, Summaries, JPL Publ. 91-28, 43-50.

Roberts D.A., Smith M.O., Sabol D.E., Adams J.B., Ustin S. 1992 Mapping the spectral variability in photosytetic and nonphotosynthetic vegetation, soils and shade using AVIRIS. In Annual JPL Airborne Geoscience Workshop, 3, Pasadena, CA, Summaries, JPL Publ. 92-14, 38-40.

Roberts D.A., Smith M.O., Adams J.B. 1993a. Green vegetation, nonphotosynthetic vegetation, and soil in AVIRIS data. Remote Sens. Environ., 44:255-269.

Roberts D.A., Green R.O., Sabol D.E., Adams J.B. 1993b. Temporal changes I Endmember abundances, liquid water and water vapor over vegetation at Jasper Ridge. In: JPL Airborne Earth Science Workshop, 4, Pasadena, CA, Summaries, JPL Publ. 93-96, 153-156

Roberts D.A., Gardner M., Church R., Ustin S., Scheer G., Green R.O. 1998a. Mapping chaparral in the Santa Monica Mountains using multiple endmember spectral mixture models. Remote Sens. Environ., 65:267-279.

Roberts D.A., Gardner M., Regelbrugge J., Pedreros D., Ustin S.L. 1998b. Mapping the distribution of wildfire fuels using AVIRIS in the Santa Monica Mountains. In: JPL Airborne Earth Science Workshop, 7, Pasadena, CA, Summaries, JPL Publ. 97-21, p.345-352

Sabol D.E. Jr., Adams J.B., Smith M.O. 1990. Predicting the spectral detectability of surface materials using spectral mixture analysis. In: IEEE International Geoscience Remote Sensing Syposium '90, Proceedings 2, 967-970.

Sabol D.E. Jr., Adams J.B., Smith M.O., Gillespie A.R. 1991. Target detection thresholds using imaging spectrometer data. In: Annual JPL Airborne Visible/Infrared Imaging Spectrometer (AVIRIS) Workshop, 3, Pasadena, CA, Summaries, JPL Publ. 91-28, p.99-108.

Sabol D.E. Jr., Adams J.B., Smith M.O. 1992a. Quantitative subpixel spectral of targets in multiespectral images, J. Geophys. Res., 97: 2659-2672.
Sabol D.E. Jr., Roberts D.A., Smith M.O., Adams J.B. 1992b. Temporal variation in spectral detection thresholds of substrate and vegetation in AVIRIS images. In: Annual JPL Airborne Geoscience Workshop, 3, Summaries, JPL Publ. 92-14, 32-134.

Sabol D.E. Jr., Roberts D.A., Adams J.B., Smith M.O. 1993. Mapping and monitoring changes in vegetation communities of Jasper Ridge, CA, Using Spectral Fractions Derived From AVIRIS Images. In: JPL Airborne Earth Science Workshop, 4, Pasadena, CA, Summaries, JPL Publ. 93-96, 157-160.

Sherman D.M. \& Waite T.D. 1985. Electronic spectra of Fe3+ oxides and oxide hidroxides in the near IR to near UV. Am. Mineral, 70:1262-1269.

Singer R. \& McCord T.B. 1979. Mars: large scale mixing of bright and dark surface materials and implication for analysis of spectral reflectance. In: Lunar Planet Sci. Conf., 10, Proceedings, 1835-1848.

Smith M.O. \& Adams J.B. 1985a. Strategy for analyzing mixed pixels in remotely sensed imagery. In: NASA/JPL Aircraff SAR Workshop, Pasadena, CA, Proceedings, JPL Pub.85-39, 47-48.

Smith M.O. \& Adams J.B. 1985b. Interpretation of AIS images of Cuprite, Nevada, using constrains of spectral mixtures. In: Airborne Imaging Spectrometer Data Analysis Workshop, Pasadena, CA, Proceedings, JPL Publ. 85-41, 62-68.

Smith M.O., Johnson P.E., Adams J.B. 1985. Quantitative determination of mineral types and anbundances from reflectance spectra using principal components analysis. Proc. $15^{\text {th }}$ Lunar Planet. Sci. Conf. Part2. J Geophys. Res., 90, Suppl., p. C797-C804.

Smith M.O., Roberts D.A., Shipman H.M., Adams J.B., Wills S.C., Gillespie A.R. 1987. Calibrating AIS images using the surface as reference. In: Airborne Imaging Spectrometer Workshop, 3, Proceedings, JPL Publ. 87-30, 10p.

Smith M.O., Adams J.B., Ustin S.L., Roberts D.A. 1992. Using endmembers in AVIRIS images to estimate changes in vegetative biomass. In: Annual JPL Airborne Geoscience Workshop, 3, Pasadena, CA, Summaries, JPL Publ. 92-14, 69-71.

Smith M.O., Roberts A.D., Hill J., Mehl W., Hosgood B., Verdebout J., Schmuck G., Koechler C., Adams J. 1994. A new approach to quantifying abundances of materials in multispectral images. In: IGARSS 1994, Proceedings, 2372-2674.

Staid M.I., Pieters C.M., Head J.W. 1996. Mare tranquilitatis: basalt emplacement history and relation to lunar samples, J. Geophys. Res., 101:23213-23228.

Towensend T.E. 1987. Discrimination of iron alteration minerals in visible and near-infrared data. J Geophys. Res., 92(B2):1441-1454.

Trescasses J.J., Melfi A.J., Oliveira S.M.B. 1980, Nickeliferous laterites of Brazil. In: International Seminar on Laterisation Process. Trivandrum, Índia, Proceeding, 170-184.

Vane G. \& Goetz A.F.H. 1988. Terrestrial imaging spectrometry, Remote Sens. Environ., 24:1-29

Vane G., Chrisp M., Enmark H., Macenka S., Solomon J. 1984. Airborne Visible/Infrared Imaging Spectrometer (AVIRIS): an advanced tool for earth remote sensing. In: IEEE Int. Geosci. Remote Sensing Symp., IEEE, New York, Proceedings, SP215, 751-757.

Manuscrito A-1325

Recebido em 06 de março de 2002

Revisão dos autores em 05 de março de 2003 Reevisão aceita em 10 de março de 2003 